\title{
Definição de regiões de interesse em problemas multiobjetivo utilizando hiper-elipses
}

\author{
Patrick P. F. Ferreira ${ }^{1}$, Luciana B. Cosme ${ }^{2}$, Allysson S. M. Lacerda ${ }^{3}$ \\ ${ }^{1}$ Programa de Pós-Graduação em Modelagem Computacional de Sistemas (PPGMCS) \\ Universidade Estadual de Montes Claros (Unimontes) \\ Caixa Postal 39.401-089 - Montes Claros - MG - Brasil \\ ${ }^{2}$ IFNMG Campus Montes Claros \\ Rua Dois, 300, Village do Lago I, 39404-058, Montes Claros - MG \\ ${ }^{3}$ Departamento de Ciências da Computação - DCC \\ Universidade Estadual de Montes Claros (Unimontes) \\ Caixa Postal 39.401-089 - Montes Claros - MG - Brasil
}

\begin{abstract}
This paper presents a new methodology to incorporate preferences in multiobjective problems through the definition of an ellipsoid-shapped region of interest. This new layer aplied to the optimization algorithm aims to help the decision making, as the method shows solutions according to the defined preferences.Futhermore, the ellipsoid shape allows the user to change the quantity of selected elements by changing the ellipsoid focus, expanding or shrinking the coverage area of the Pareto frontier.
\end{abstract}

Resumo. Este artigo apresenta uma nova metodologia de incorporação de preferências em problemas multiobjetivos através da definição de uma região de interesse na forma de uma hiper-elipse. Essa camada adicionada ao algoritmo de otimização visa auxiliar a tomada de decisão, pois o método mostra soluções de acordo com as preferências definidas. Além disso, a forma hiper-elipsoide permite que o usuário altere a quantidade de elementos selecionados ao se modificar o foco do hiper-elipsoide, ampliando ou reduzindo assim a área de cobertura da fronteira Pareto.

\section{Introdução}

Durante o processo de tomada de decisões é comum se deparar com problemas em que se deve encontrar a melhor maneira de alocar recursos limitados para a melhoria de um processo; a estes problemas, damos o nome de problemas de otimização. De uma maneira simplificada, podemos dizer que esta categoria de problemas se baseia em encontrar os pontos ótimos - máximos ou mínimos, dependendo da necessidade expressada pelo problema - para uma ou mais funções objetivo respeitando suas restrições.

[Coello et al. 2007] aponta que, apesar da variedade de técnicas desenvolvidas na área de otimização, a complexidade dos problemas com múltiplos objetivos requer abordagens diferenciadas - ainda mais ao considerar que tais problemas possuem objetivos conflitantes e, com isso, melhorar um objetivo implica em piorar algum outro; para estes casos, o problema apresenta um conjunto de soluções ótimas (fronteira Pareto) ao invés de uma única solução. 
Porém, o processo de tomada de decisão não termina quando se encontra a fronteira Pareto para um problema multiobjetivo; o tomador de decisão ainda precisa definir, dentro daquele conjunto de pontos ótimos, qual melhor se adéqua a suas preferências o que não é uma tarefa trivial [Coello et al. 2007]. Neste contexto, a incorporação de preferências através da definição de regiões de interesse surge como uma alternativa para filtrar, dentre um conjunto de soluções ótimas, aquelas que melhor se adequam ao desejado pelo tomador de decisão.

Dentre as propostas disponíveis na literatura para incorporação de preferências, destacam-se os métodos que definem apenas um ponto de interesse ([Qi et al. 2019] e [Filatovas et al. 2020]), que é atualizado ao longo da execução do algoritmo. Outra abordagem, proposta por [Meneghini et al. 2021], utiliza hipercones para ajustar os vetores de pesos em métodos baseados em decomposição, mas é restrita a esta classe de algoritmos. No presente trabalho, é apresentado um novo método de incorporação de preferências baseado em hiper-elipses que permite ajustar a pressão de seleção de forma simples e pode ser utilizado em qualquer classe de algoritmos.

Este artigo está estruturado da seguinte forma: na seção 1 é apresentada a introdução; na seção 2 é apresentada a fundamentação teórica, onde são demonstrados conceitos importantes para o entendimento a respeito da incorporação de preferências em problemas multiobjetivo - tais como o que são problemas multiobjetivo, fronteira Pareto ou incorporação de preferência; a seção 3 descreve os métodos utilizados para alcançar o objetivo da pesquisa; na seção 4 é feita uma análise sobre os resultados obtidos pelo algoritmo, comparando os resultados obtidos ao utilizar o operador de incorporação de preferências na forma de hiper-elipsoide com os resultados dos algoritmos originais; a seção 5 é descrito o que se pode concluir sobre os resultados encontrados e o futuro da pesquisa.

\section{Fundamentação Teórica}

\subsection{Otimização Multiobjetivo}

Segundo [Santos et al. 2019], quando tratamos de otimização no mundo real, podem existir objetivos que muitas vezes são conflitantes. Um exemplo de problema desse tipo pode ser analisado na tarefa de comprar um carro: o desejado - ou o ponto ótimo para o nosso problema - é aquela solução que fornece menor custo, mas aumenta o conforto ou apresenta uma maior eficiência energética (utilizando menos combustível); carros mais confortáveis ou mais econômicos podem custar mais caro do que um carro sem essas vantagens. De maneira formal, [Deb 2001] descreve problemas de otimização multiobjetivo da seguinte forma:

$$
\begin{array}{rrr}
\text { minimizar/maximizar } & f_{m}(x) & m=1,2,3, \ldots, M ; \\
\text { restrito a } & g_{j}(x) \geq 0, & j=1,2,3, \ldots, J ; \\
& h_{k}(x)=0, & k=1,2,3, \ldots, K \\
& x_{i}^{(L)} \leq x_{i} \leq x_{i}^{(U)} & i=1,2,3, \ldots, n
\end{array}
$$

em que: 
$M$ : quantidade de objetivos do problema;

$J$ : quantidade de restrições de desigualdades;

$K$ : quantidade de restrições de igualdade;

$n$ : quantidade de restrições de limites;

Para avaliar as soluções possíveis para problemas de otimização multiobjetivo, é utilizado o conceito de dominância Pareto, que determina qual, dentre as soluções encontradas, apresenta um melhor resultado. Esse conceito diz que, dados dois vetores de decisão $x, y \in F$, em que $F$ é o conjunto de soluções factíveis, pode-se dizer que a solução $x$ domina a solução $y$ em um problema de minimização se $f_{i}(x) \leq f_{i}(y)$ e, em um problema de maximização, se $f_{i}(x) \geq f_{i}(y)$ - ou seja, a solução apresenta resultado melhor em pelo menos um dos objetivos e não é pior para os demais. Desta forma, teremos um conjunto de pontos Pareto-ótimos (ou Pareto-eficientes), como os demonstrados na Figura 1 - em que a linha e os pontos em azul representam as soluções não-dominadas e os pontos em vermelho, as dominadas; observe que nenhum ponto azul tem menor valor que o outro em pelo menos um objetivo, e que são sempre menores que os pontos em vermelho em pelo menos um objetivo.

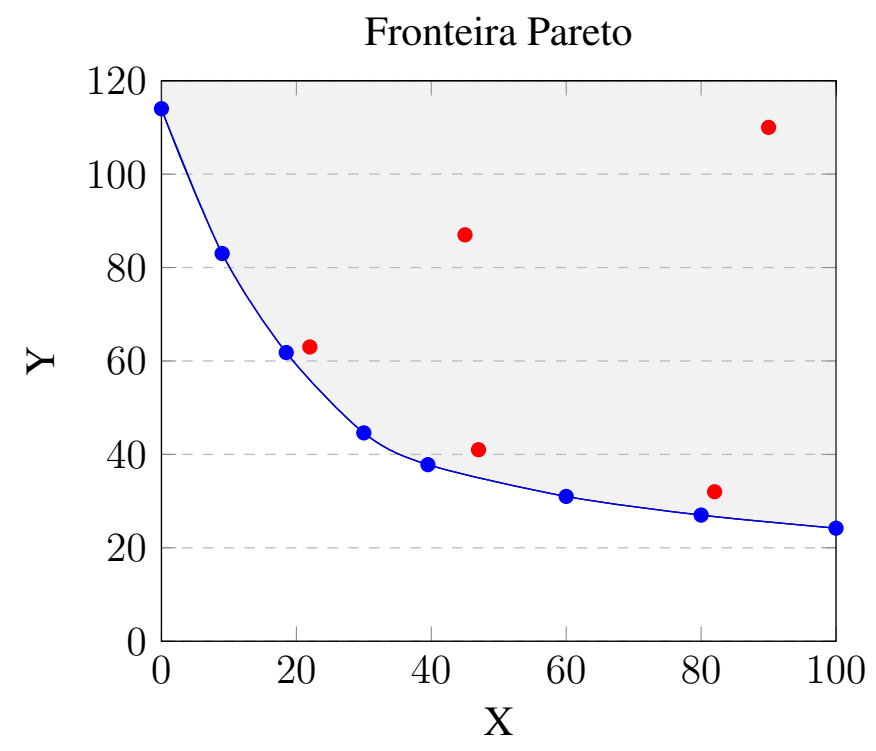

Figura 1. Fronteira Pareto

A fronteira Pareto-ótimo pode ser vista como o conjunto dos melhores resultados possíveis para um problema de otimização multiobjetivo. De uma forma simplificada, pode-se dizer que este conjunto define o limite de até onde os resultados podem ser melhorados para um objetivo sem que haja a piora de outro.

\subsection{Incorporação de preferências}

Como dito anteriormente, o processo de tomada de decisão não termina quando se encontra a fronteira Pareto de um problema multiobjetivo. Após a definição das soluções Pareto-eficientes, ainda é preciso decidir qual delas será aplicada.

A busca por metodologias que facilitem a identificação das soluções que melhor se adequem ao que o tomador de decisão deseja levou à criação de operadores para incorporação de preferências para algoritmos multiobjetivo. Segundo 
[Bechikh et al. 2015], essas preferências são usadas para guiar a busca a uma determinada parte da fronteira Pareto - a região de interesse (ROI - Region of Interest). A incorporação de preferências utilizando esse tipo de metodologia pode ser vista na Figura 2, onde a Figura 2a mostra os pontos encontrados pelo algoritmo na fronteira Pareto e a própria fronteira Pareto (a linha em azul) e a Figura $2 b$ demonstra os pontos obtidos utilizando a incorporação de preferências, a região de interesse do tomador de decisão (em verde) e a fronteira Pareto (em azul). Note que, em $2 \mathrm{~b}$ a busca é guiada para encontrar pontos somente na região de interesse.

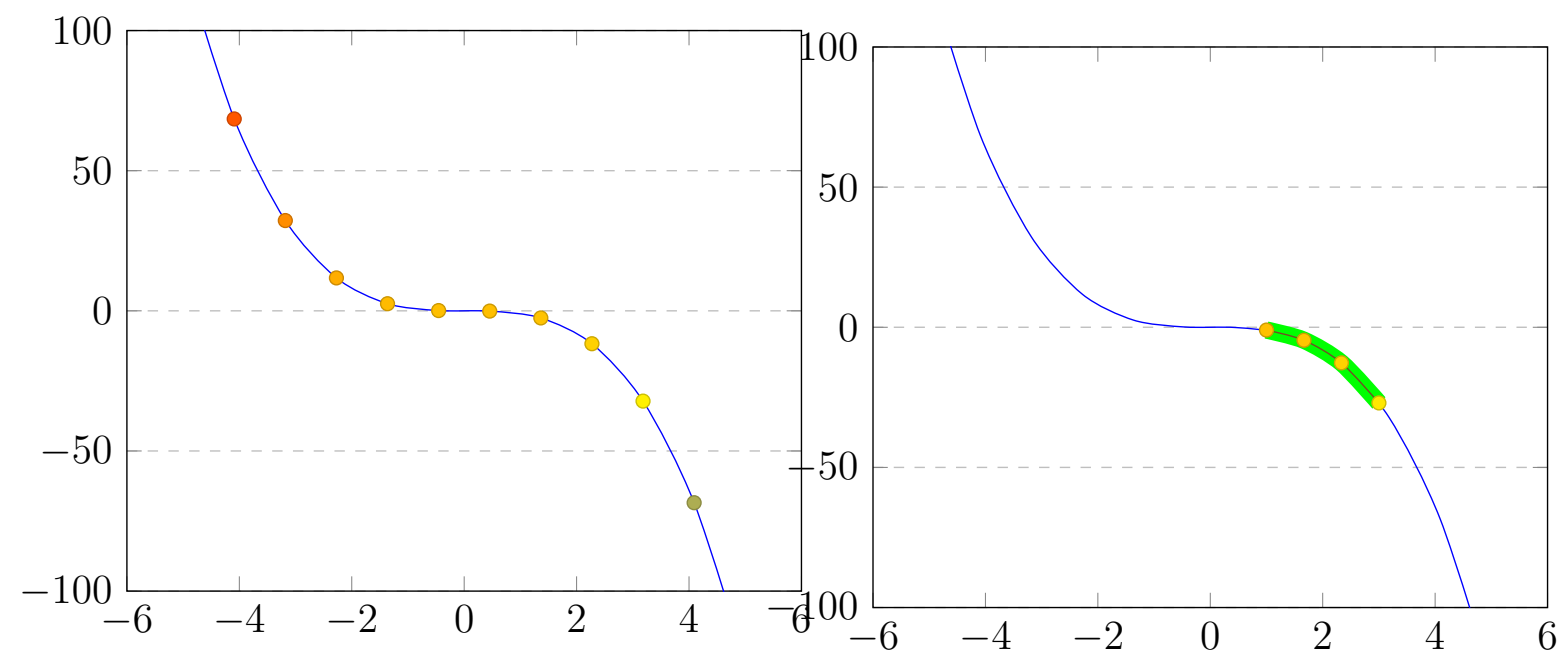

(a) Resultado de um algoritmo multiobjetivo sem incorporação de preferências

(b) Resultado utilizando incorporação de preferências

Figura 2. Definição da região de interesse da fronteira Pareto

Diferentes tipos de metodologia vêm sendo utilizadas para a definição dessa região de interesse em algoritmos multiobjetivos. [Bechikh et al. 2015] divide as soluções utilizadas para incorporação de preferências nas seguintes categorias:

- Abordagens baseadas em pesos: apresentam diferentes métodos que permitem agregar uma importância à solução na forma de peso. Os trabalhos propostos por [Deb 2003], que adiciona um peso ao cálculo da distância euclidiana do algoritmo NSGA-II, ou a proposta de [Branke and Deb 2005], em que o peso é adicionado no calculo de Crownding Distance, são dois exemplos deste tipo de proposta.

- Abordagens baseadas na classificação de soluções: buscam utilizar métodos nos quais o tomador de decisões classifica quais soluções estão de acordo com suas preferências. Um exemplo desse tipo de abordagem é o trabalho realizado por [Deb et al. 2010] no qual, após um conjunto de soluções próximas a fronteira Pareto-ótimo ser encontrado, é requisitado ao tomador de decisões que classifique as soluções de acordo com suas preferências.

- Abordagens baseadas na classificação de objetivos: utilizam uma metodologia na qual o tomador de decisões classifica quais objetivos estão de acordo com suas preferências. A da proposta de [Rachmawati and Srinivasan 2010], em que o tomador de decisões classifica, a priori, os objetivos de acordo com suas preferências é um exemplo desse tipo de metodologia.

- Abordagens baseadas em Trade-Off: esta metodologia permite ao tomador de decisão definir o trade-off máximo aceitável para os objetivos - ou seja, o quanto 
o tomador de decisão está disposto a sacrificar daquele objetivo em prol de outro. A proposta de [Branke et al. 2001] é um exemplo desse tipo de metodologia, em que o tomador de decisão deve definir a priori, para cada par de objetivos, o tradeoff máximo aceitável.

- Abordagens baseadas em Desirability Functions: utilizam funções de desejabilidade e se baseiam em considerar, segundo [Costa et al. 2011], as características de qualidade interessantes como uma função. O trabalho proposto por [Wagner and Trautmann 2010] é um exemplo desse tipo de metodologia, buscando utilizar funções objetivo de desejabilidade (por serem otimizadas) no lugar das funções objetivo do problema.

- Abordagens baseadas em ponto de referência: utilizam pontos de referência definidos a priori para indicar a região da fronteira Pareto mais interessantes para o tomador de decisão. Uma vantagem dessa metodologia é que só é preciso a definição de um ponto para que se encontre a região de interesse, ao contrário das abordagens baseadas em classificação de objetivos, de soluções e de pesos. Os trabalhos nessa área envolvem os já citados: [Meneghini et al. 2021], em que é definido um cone de interesse; [Qi et al. 2019] e [Filatovas et al. 2020], nos quais a região é definida por um ponto utópico.

Este trabalho faz parte das abordagens baseadas em ponto de referência, utilizando dois pontos de referência para criar uma hiper-elipse de preferência. Esta categoria se mostrou interessante pela facilidade para o tomador de decisão definir uma região de interesse - ou seja, ele precisa somente definir qual o ponto utópico ou de referência.

\section{Metodologia}

O método proposto utiliza uma hiper-elipse para a definição de uma região de interesse a partir de dois pontos referência definidos a priori pelo tomador de decisão. A forma elipsoidal foi escolhida por permitir alterar a pressão de seleção sobre os resultados modificando o a distância focal da elipse, como demonstrado na Figura 3, em que as elipses $3 a$ e $3 b$ apresentam os mesmos pontos em suas extremidades, mas a distância focal da elipse $3 b$ é menor que a distância focal da elipse $3 a$, o que pode possibilitar que mais valores da fronteira Pareto sejam selecionados. No entanto, saber o valor exato do foco desejado pode não ser tão simples, uma vez que a escala do hiper-elipsoide pode variar de acordo com o problema. Desta forma, para melhor auxiliar o tomador de decisões, foi definido o foco como estando a um determinado percentual de distância, escolhido pelo tomador de decisão, partindo dos pontos extremos em direção ao centro do hiperelipsoide - ou seja, utilizando essa metodologia, quanto maior a distância percentual dos foco aos extremos, menor será o valor da distância focal e maior serão os demais raios do hiper-elipsoide. Os raios em todas as demais dimensões foram definidas com o mesmo valor.

Matematicamente, a partir do exposto por [Tee 2005], pode-se definir a equação geral de um hiper-elipsoide como descrito nas Equações (5), (6) e (7), em que (5) demonstra a equação para duas dimensões, (6) para três dimensões e (7) apresenta a generalização para $d$ dimensões, onde $-c_{n}$ representa a movimentação do centro do hiper-elipsoide no plano cartesiano - sem ele, o centro do hiper-elipsoide é definido na origem. Desta forma, pode-se dizer que quando o resultado da equação é igual a 1, o ponto está sobre a curva do 


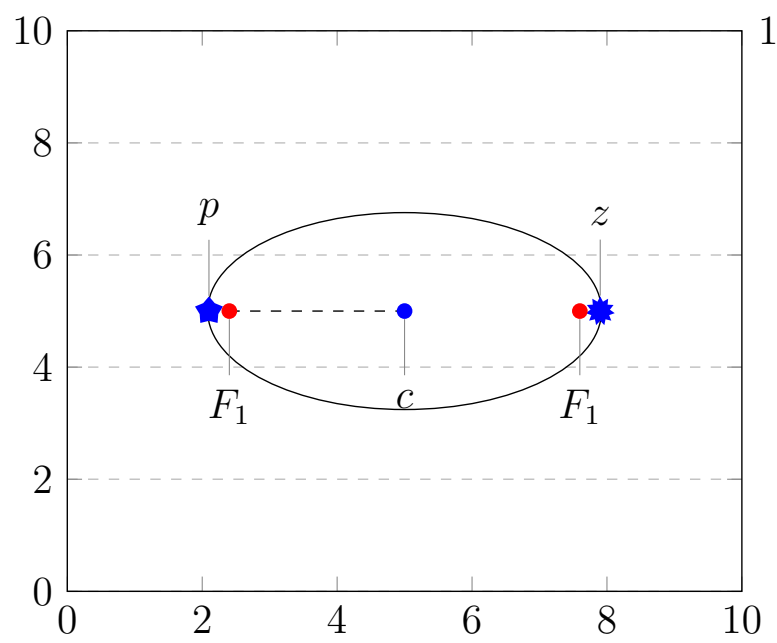

(a) Elipse A

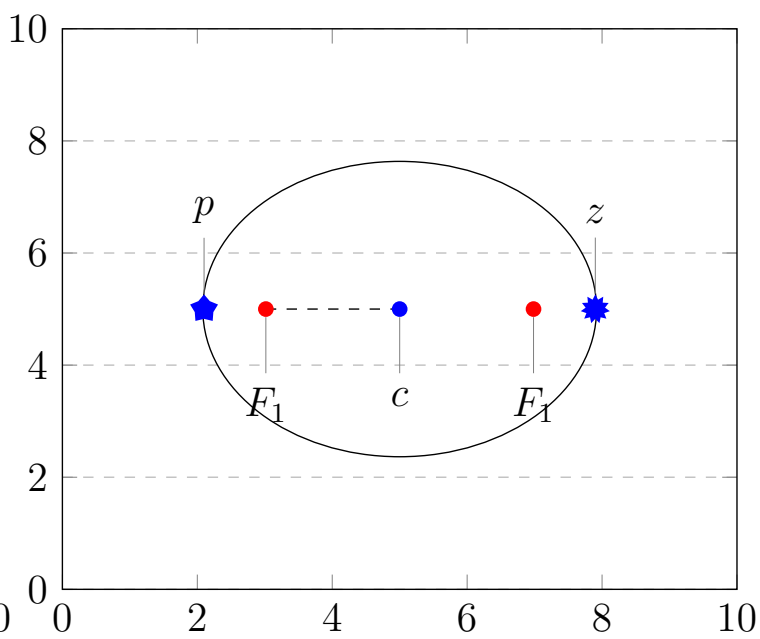

(b) Elipse B

Figura 3. Alteração de pressão de seleção em uma elipse

hiper-elipsoide; quando este valor é menor que 1, o ponto está dentro do hiper-elipsoide e; quando o valor é maior que 1 , o ponto está fora do hiper-elipsoide.

$$
\begin{gathered}
\frac{\left(x_{1}-c_{1}\right)^{2}}{r_{1}^{2}}+\frac{\left(x_{2}-c_{2}\right)^{2}}{r_{2}^{2}}=1 \\
\frac{\left(x_{1}-c_{1}\right)^{2}}{r_{1}^{2}}+\frac{\left(x_{2}-c_{2}\right)^{2}}{r_{2}^{2}}+\frac{\left(x_{3}-c_{3}\right)^{2}}{r_{3}^{2}}=1 \\
\cdot \\
\cdot \\
\cdot \\
\sum_{n=1}^{d} \frac{\left(x_{n}-c_{n}\right)^{2}}{r_{n}^{2}}=1
\end{gathered}
$$

em que:

$d$ : número de dimensões;

$x$ : coordenada do ponto a ser verificado;

$r$ : raio da elipse na determinada dimensão;

$c$ : coordenada do centro da hiper-elipse na dimensão.

Porém, como visualizado na Figura 3, as Equações demonstradas em (5), (6) e (7) definem sempre um hiper-elipsoide sem inclinação - os ângulos dos eixos da elipse em relação aos eixos das abcissas e ordenadas vão ser sempre os mesmos. Para solucionar essa questão, é necessário aplicar algumas transformações geométricas a este hiper-elipsoide ou aos pontos analisados. A Figura 4 demonstra a formação de um hiperelipsoide dados os pontos $p$ e $z$ em relação a fronteira Pareto; note que somente a região da fronteira dentro do hiper-elipsoide será selecionada e que ambos os hiper-elipsoides apresentam os mesmos pontos $p$ e $z$, mas com distâncias focais diferentes. 


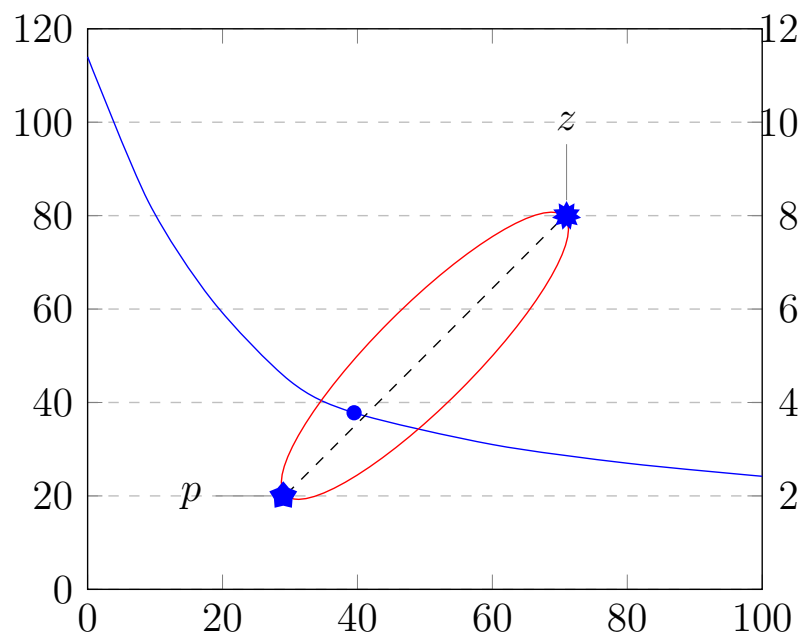

(a) Elipse A

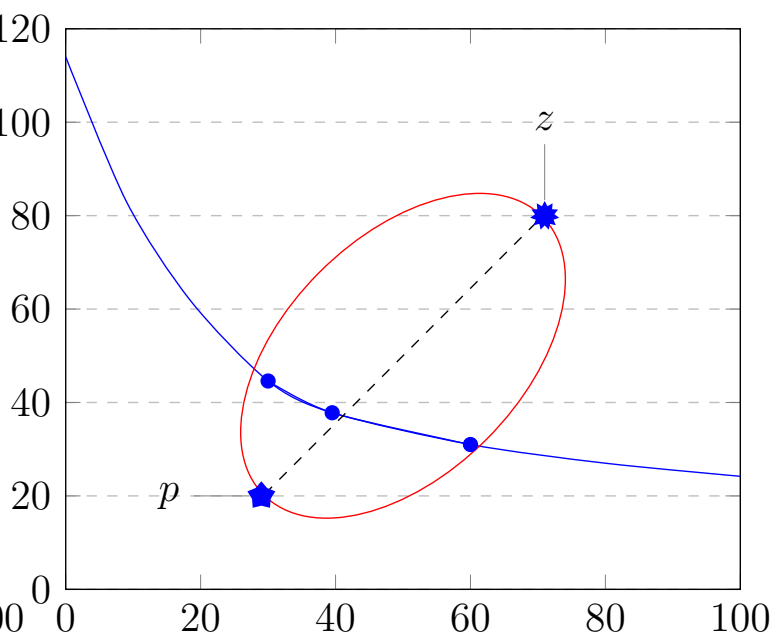

(b) Elipse B

Figura 4. Elipses rotacionadas em relação à fronteira Pareto

Segundo [Wolbert 1988], a base de uma transformação geométrica é o mapeamento de um sistema de coordenadas em outro utilizando, geralmente, series de multiplicações de matrizes (Equação 12). O primeiro passo para realizar a rotação é definir um ponto fixo em torno do qual a rotação irá ser efetuada - centro de rotação, $p=\left(p_{x}, p_{y}, p_{z}\right)$. A partir deste ponto, o próximo passo será efetuar uma translação dos elementos de forma que o centro de rotação fique posicionado na origem (Equação 8) para só então realizar a rotação em torno do eixo $x$ (Equação 9), seguida pela rotação em torno do eixo $y$ (Equação 10) e, finalmente, em torno do eixo $z$ (Equação 11). O último passo do processo é fazer uma nova translação em $-p$ para que os pontos voltem para a localização original.

$$
\begin{gathered}
T(p)=\left(\begin{array}{l}
p_{x} \\
p_{y} \\
p_{z}
\end{array}\right) \\
R_{x}(\theta)=\left(\begin{array}{ccc}
1 & 0 & 0 \\
0 & \cos \theta & -\sin \theta \\
0 & \sin \theta & \cos \theta
\end{array}\right) \\
R_{y}(\theta)=\left(\begin{array}{ccc}
\cos \theta & 0 & \sin \theta \\
0 & 1 & 0 \\
-\sin \theta & 0 & \cos \theta
\end{array}\right) \\
R_{z}(\theta)=\left(\begin{array}{ccc}
\cos \theta & \sin \theta & 0 \\
-\sin \theta & \cos \theta & 0 \\
0 & 0 & 1
\end{array}\right) \\
M=T(p) \times R_{x}(\theta) \times R_{y}(\theta) \times R_{z}(\theta) \times T(-p)
\end{gathered}
$$


Este método de rotação apresenta um conjunto de matrizes diferentes para cada conjunto de dimensões. Desta maneira, para rotações em duas dimensões é utilizada uma matriz de rotação, pois se trata basicamente de uma rotação em torno do eixo Z; para três dimensões, serão 3 matrizes de rotação (uma em torno de cada eixo) e assim sucessivamente. A busca por um método generalista de rotação levou ao algoritmo Aguilera-Perez [Aguilera and Pérez-Aguila 2004].

\subsection{Algoritmo Aguilera-Perez adaptado}

O algoritmo proposto por [Aguilera and Pérez-Aguila 2004] busca gerar matrizes para rotações de vetores n-dimensionais em espaços euclidianos. Segundo o autor, podemos considerar uma rotação $n$-dimensional como uma rotação em torno de um sub-espaço $(n-2)$-dimensional. Desta forma, com uma série de rotações pode-se atingir o desejado. O algoritmo de rotação de Aguilera-Perez, porém, leva em consideração que a rotação a ser efetuada seja do mesmo ângulo em todas as dimensões; para adaptá-lo a proposta deste trabalho, isto foi alterado para que o algoritmo receba o ponto médio do hiper-elipsoide $\left(p_{\text {medio }}\right)$, assim como seus extremos $(p$ e $z)$ e a quantidade de dimensões $(d)$ e calcule o angulo dinamicamente para cada par de dimensões ao invés de utilizar um angulo único (Algoritmo 1).

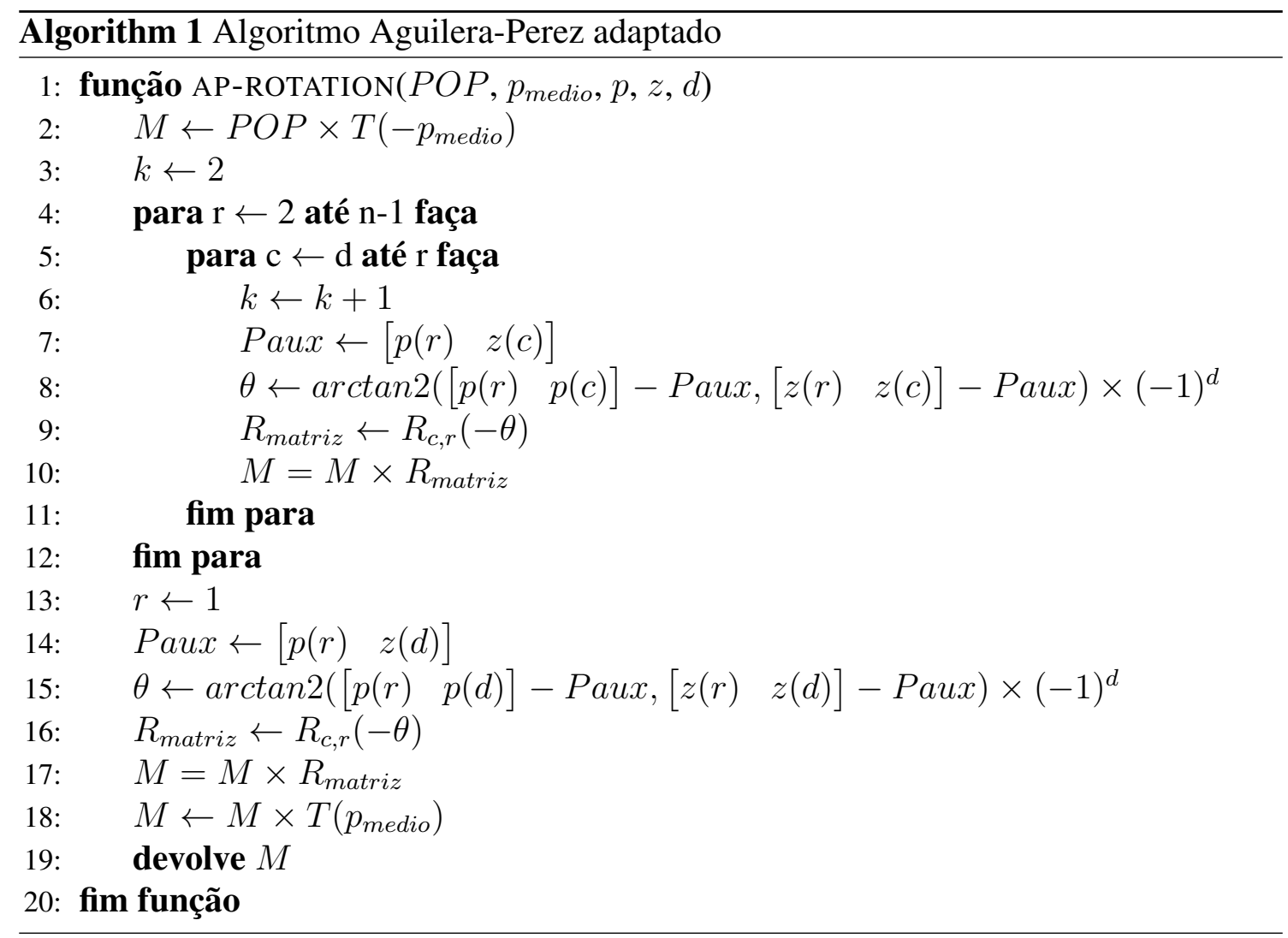

Como descrito anteriormente, o primeiro passo para efetuação da rotação é a translação para o novo centro de rotação. Para este trabalho, usou-se o ponto médio do hiper-elipsoide $\left(p_{\text {medio }}\right)$ como centro de rotação. A geração da matriz de rotação n-dimensional utilizada por [Aguilera and Pérez-Aguila 2004] foi descrito por [Duffin and Barrett 1994], e pode ser vista descrita na Equação (13). 


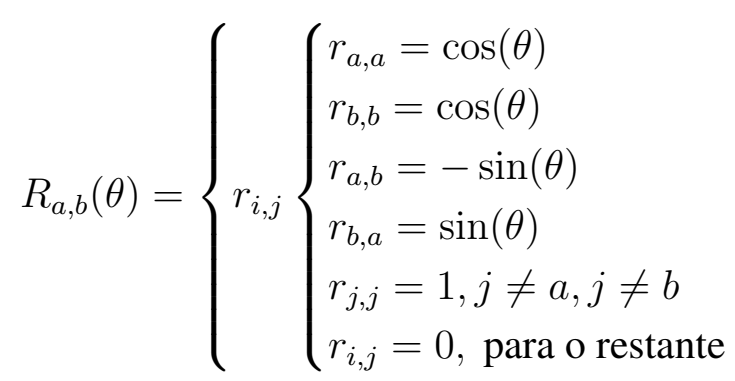

\subsection{Estrutura do algoritmo}

Utilizando os conceitos descritos anteriormente, podemos agora realizar a incorporação de preferências utilizando um hiper-elipsoide. A hiper-elipse é formada por dois pontos ( $p$ e $z$ ) de referência, até então, escolhidos pelo tomador de decisão. De uma maneira simplificada, pode-se definir a metodologia aplicada de acordo com os seguintes passos:

1. A partir dos pontos $p$ e $z$ (os dois extremos da nossa elipse), são encontrados o ponto médio e os focos do hiper-elipsoide;

2. Utilizando o método de Aguilera-Perez, todas as soluções factíveis são rotacionadas na direção contrária a do hiper-elipsoide $(-\theta)$;

3. Utilizando a função do hiper-elipsoide (equação 7), são encontrados os pontos que estão contidos dentro do mesmo - onde o resultado da equação é menor ou igual a 1 ;

4. Os pontos dentro do hiper-elipsoide são selecionados, o restante é descartado.

\section{Resultados}

Para investigar a performance da solução apresentada, foram utilizados o hipervolume (HV), por ajudar a medir a cobertura e a distância geracional invertida (IGD) para medir a convergência para a fronteira Pareto. As duas métricas foram escolhidas pois o HV possibilita medir o quanto da fronteira Pareto está sendo selecionada (quanto maior o HV, maior a área de cobertura da fronteira pareto); e o IGD, que ajuda a medir a qualidade dos pontos encontrados em relação aos pontos da fronteira Pareto. Essas escolhas foram feitas com o intuito de determinar se o algoritmo teve alguma melhora de desempenho utilizando o operador em relação a cobertura e proximidade da fronteira Pareto. Além disso, foram efetuadas 100 execuções para os problemas DTLZ1 e DTLZ2 - parte do conjunto de problemas multiobjetivos propostos por [Deb et al. 2002] - com 3 dimensões e os pontos $p$ e $z$ dispostos como mostrado na Tabela 1. Os pontos foram escolhidos de modo que o ponto $p$ ficasse acima da fronteira Pareto e o ponto $z$ abaixo - vale ressaltar que estes dois pontos são definidos a priori pelo tomador de decisão e que os pontos utilizados aqui foram escolhidos arbitrariamente.

\begin{tabular}{||c|c|c||}
\hline Problema & $p$ & $z$ \\
\hline DTLZ1 & $(0.4,0.38,0.4)$ & $(0.1,0.1,0.1)$ \\
DTLZ2 & $(0.9,0.88,0.75)$ & $(0.4,0.33,0.37)$ \\
\hline
\end{tabular}

Tabela 1. Pontos $p$ e $z$ utilizados para os problemas DTLZ1 e DTLZ2

As médias e desvios-padrão do hiper-volume (HV) e da distância geracional invertida (IGD) dos resultados obtidos, considerando-se 100 execuções, com os algoritmos 
dentro da região de interesse para os problemas DTLZ1 e DTLZ2 podem ser vistos, respectivamente, nas Tabelas 2 e 3. Para o problema DTLZ1, pode-se notar que os algoritmos que utilizam o operador descrito neste trabalho apresentaram, para o AGE-II, NSGA-II e NSGA-II-DE resultados próximos aos obtidos dentro da região de interesse se comparado ao algoritmo original (sem a incorporação de preferências). Deve-se notar também que o algoritmo foi capaz de utilizar o operador com sucesso para gerar pontos dentro da região de interesse definida pelos extremos do hiper-elipsoide.

\begin{tabular}{||c|c|c|c||}
\hline \multirow{2}{*}{ Algoritmo } & \multirow{2}{*}{ Método } & \multicolumn{2}{|c||}{ DTLZ1 } \\
\cline { 3 - 4 } & & HV & IGD \\
\hline \multirow{2}{*}{ AGE-II } & Original & $0.1645 \pm 0.2545$ & $0.5398 \pm 0.4649$ \\
\cline { 2 - 4 } & Hiper-elipsoide & $0.1323 \pm 0.2171$ & $0.5119 \pm 0.3395$ \\
\hline \multirow{2}{*}{ ANSGA-III } & Original & $0.3948 \pm 0.3288$ & $0.2565 \pm 0.2405$ \\
\cline { 2 - 4 } & Hiper-elipsoide & $0.2311 \pm 0.2442$ & $0.3286 \pm 0.2349$ \\
\hline \multirow{2}{*}{ NSGA-II } & Original & $0.4359 \pm 0.3426$ & $0.2463 \pm 0.2941$ \\
\cline { 2 - 4 } & Hiper-elipsoide & $0.3236 \pm 0.2677$ & $0.2509 \pm 0.1764$ \\
\hline \multirow{2}{*}{ NSGA-II-DE } & Original & $0.0253 \pm 0.1210$ & $2.7639 \pm 1.7928$ \\
\cline { 2 - 4 } & Hiper-elipsoide & $0.0272 \pm 0.0923$ & $2.6416 \pm 1.8264$ \\
\hline \multirow{2}{*}{ NSGA-III } & Original & $0.4531 \pm 0.3144$ & $0.1964 \pm 0.1765$ \\
\cline { 2 - 4 } & Hiper-elipsoide & $0.2827 \pm 0.2521$ & $0.2784 \pm 0.1936$ \\
\hline
\end{tabular}

Tabela 2. Análise comparativa, considerando média e desvio padrão em 100 execuções, entre a solução apresentada e o algoritmo sem incorporação de preferências com o problema DTLZ1

Apesar dos resultados para o problema DTLZ2 não terem sido tão próximos quanto os do DTLZ1, o algoritmo ainda obteve sucesso na geração de pontos somente dentro da região de interesse - obtendo um IGD médio um pouco melhor para o NSGAIII, para os pontos dentro do hiper-elipsoide.

\begin{tabular}{||c|c|c|c||}
\hline \multirow{2}{*}{ Algoritmo } & Método & \multicolumn{2}{|c||}{ DTLZ2 } \\
\cline { 3 - 4 } & & HV & IGD \\
\hline \multirow{2}{*}{ AGE-II } & Original & $0.3643 \pm 0.1089$ & $0.2609 \pm 0.1042$ \\
\cline { 2 - 4 } & Hiper-elipsoide & $0.1676 \pm 0.0044$ & $0.4482 \pm 0.0069$ \\
\hline \multirow{2}{*}{ ANSGA-III } & Original & $0.4724 \pm 0.0988$ & $0.1287 \pm 0.0972$ \\
\cline { 2 - 4 } & Hiper-elipsoide & $0.1548 \pm 0.0140$ & $0.4643 \pm 0.0227$ \\
\hline \multirow{2}{*}{ NSGA-II } & Original & $0.4379 \pm 0.1016$ & $0.1462 \pm 0.1019$ \\
\cline { 2 - 4 } & Hiper-elipsoide & $0.1668 \pm 0.0049$ & $0.4469 \pm 0.0067$ \\
\hline \multirow{2}{*}{ NSGA-II-DE } & Original & $0.3900 \pm 0.0856$ & $0.1640 \pm 0.0878$ \\
\cline { 2 - 4 } & Hiper-elipsoide & $0.1665 \pm 0.0043$ & $0.4456 \pm 0.0074$ \\
\hline \multirow{2}{*}{ NSGA-III } & Original & $0.4813 \pm 0.0935$ & $0.4813 \pm 0.0935$ \\
\cline { 2 - 4 } & Hiper-elipsoide & $0.1526 \pm 0.0123$ & $0.4681 \pm 0.0181$ \\
\hline
\end{tabular}

Tabela 3. Análise comparativa, considerando média e desvio padrão em 100 execuções, entre a solução apresentada e o algoritmo sem incorporação de preferências com o problema DTLZ2

Com isso, pode-se dizer que os algoritmos que fizeram o uso do operador obtiveram resultados satisfatórios - principalmente no problema DTLZ1. A utilização dos dois 
pontos $p$ e $z$ fixos provavelmente foi a causa do resultado inferior com o problema DTLZ2 - uma vez que, utilizando esta metodologia, o algoritmo pode demorar mais gerações para começar a gerar pontos dentro do hiper-elipsoide, convergindo de uma maneira menos eficiente do que o esperado para a fronteira Pareto.

\section{Considerações Finais}

Este artigo apresentou uma nova metodologia de incorporação de preferências em algoritmos de otimização multiobjetivo através da definição de uma região de interesse na forma de uma hiper-elipse. Os testes relatados neste trabalho tiveram resultados satisfatórios para problemas com 3 dimensões, mas o objetivo final dessa pesquisa será alcançar esses resultados com n-dimensões.

O trabalho apresentado, em seu estado atual, exige um conhecimento prévio a respeito do problema e da fronteira Pareto do mesmo para se definir os pontos $p$ e $z$. Para buscar melhorar a convergência e eliminar essa necessidade de conhecimento prévio da fronteira Pareto, pretende-se aplicar uma metodologia com o ponto $z$ adaptativo, de forma que a localização deste, assim como a forma do hiper-elipsoide em si, possam ser definidos considerando a distância do ponto $p$ aos demais; com isso, espera-se que existam pontos selecionados logo na primeira execução, com o raio do hiper-elipsoide diminuindo, aumentando assim a pressão de seleção, a medida que os pontos se aproximam da fronteira Pareto. Além disso, uma das propostas é que os resultados desse método de incorporação de preferência possam ser comparados com outros métodos.

\section{Referências}

Aguilera, A. and Pérez-Aguila, R. (2004). General n-dimensional rotations.

Bechikh, S., Kessentini, M., Said, L. B., and Ghédira, K. (2015). Chapter four - preference incorporation in evolutionary multiobjective optimization: A survey of the state-of-theart. volume 98 of Advances in Computers, pages 141-207. Elsevier.

Branke, J. and Deb, K. (2005). Integrating User Preferences into Evolutionary MultiObjective Optimization, pages 461-477. Springer Berlin Heidelberg, Berlin, Heidelberg.

Branke, J., Kaußler, T., and Schmeck, H. (2001). Guidance in evolutionary multiobjective optimization. Advances in Engineering Software, 32(6):499-507.

Coello, C. A. C., Lamont, G. B., and Veldhuizen, D. A. V. (2007). Evolutionary Algorithms for Solving Multi-Objective Problems. Springer-Verlag, Berlin, Heidelberg.

Costa, N. R., Lourenço, J., and Pereira, Z. L. (2011). Desirability function approach: A review and performance evaluation in adverse conditions. Chemometrics and Intelligent Laboratory Systems, 107(2):234-244.

Deb, K. (2001). Multiobjective Optimization Using Evolutionary Algorithms. Wiley, New York.

Deb, K. (2003). Multi-objective Evolutionary Algorithms: Introducing Bias Among Pareto-optimal Solutions, pages 263-292. Springer Berlin Heidelberg, Berlin, Heidelberg. 
Deb, K., Sinha, A., Korhonen, P. J., and Wallenius, J. (2010). An interactive evolutionary multiobjective optimization method based on progressively approximated value functions. IEEE Transactions on Evolutionary Computation, 14(5):723-739.

Deb, K., Thiele, L., Laumanns, M., and Zitzler, E. (2002). Scalable multi-objective optimization test problems. In Proceedings of the 2002 Congress on Evolutionary Computation. CEC'02 (Cat. No.02TH8600), volume 1, pages 825-830 vol.1.

Duffin, K. and Barrett, W. (1994). Spiders: a new user interface for rotation and visualization of n-dimensional point sets. In Proceedings Visualization '94, pages 205-211.

Filatovas, E., Kurasova, O., Redondo, J. L., and Fernández, J. (2020). A reference pointbased evolutionary algorithm for approximating regions of interest in multiobjective problems. TOP, 28:402-423.

Meneghini, I. R., Guimarães, F. G., Gaspar-Cunha, A., and Cohen, M. W. (2021). Incorporation of Region of Interest in a Decomposition-Based Multi-objective Evolutionary Algorithm, pages 35-50. Springer International Publishing, Cham.

Qi, Y., Li, X., Yu, J., and Miao, Q. (2019). User-preference based decomposition in moea/d without using an ideal point. Swarm and Evolutionary Computation, 44:597611.

Rachmawati, L. and Srinivasan, D. (2010). Incorporating the notion of relative importance of objectives in evolutionary multiobjective optimization. IEEE Transactions on Evolutionary Computation, 14(4):530-546.

Santos, W., Silva, L., and Britto, A. (2019). Incorporation of restriction treatment techniques based on the particle swarm optimization metaheuristic in the software project scheduling problem in software projects. In Anais do XVI Encontro Nacional de Inteligência Artificial e Computacional, pages 503-514, Porto Alegre, RS, Brasil. SBC.

Tee, G. (2005). Surface area and capacity of ellipsoids in n dimensions. (citr). volume 34, pages $165-198$.

Wagner, T. and Trautmann, H. (2010). Integration of preferences in hypervolume-based multiobjective evolutionary algorithms by means of desirability functions. IEEE Transactions on Evolutionary Computation, 14(5):688-701.

Wolbert, G. (1988). Geometric transformation techniques for digital images: A survey. Columbia University Computer Science Technical Reports, CUCS-390-88. Department of Computer Science, Columbia University. 